\title{
Modified Weibull analysis on banana fiber strength prediction
}

\author{
C. V. Sia ${ }^{1 *}$, L. Fernando ${ }^{1}$, A. Joseph ${ }^{2}$ and S. N. Chua ${ }^{3}$ \\ ${ }^{1}$ Mechanical Engineering Department, University of Technology (Sarawak Campus), \\ 93350 Kuching, Sarawak, Malaysia \\ *Email: cvsia@swinburne.edu.my \\ Phone: +6082260984; Fax: +6082260813 \\ ${ }^{2}$ Electronics Engineering Department, University Malaysia Sarawak, \\ 94300 Kota Samarahan, Sarawak, Malaysia \\ ${ }^{3}$ Mechanical Engineering Department, University Malaysia Sarawak, \\ 94300 Kota Samarahan, Sarawak, Malaysia
}

\begin{abstract}
Nowadays, the world is facing a crisis in regards to renewable resources and rising carbon footprint. In the microscopic scale of engineering, this is where natural fibers come into play. Natural fibers comprise of considerable advantages that include being lightweight, inexpensive and renewable in comparison to their synthetic counterparts. The fibers are being used as reinforcement in composite matrices, including hybrid composites. The main issue with natural fibers is the high disparity of its mechanical properties, especially tensile strength. In this study, Weibull statistical analysis is utilised in order to quantify the variation of the probabilistic strength of banana fibers. Single fiber tensile test was performed after the fiber diameters were measured at $1 \mathrm{~mm}$ intervals along its length. The modified Weibull distribution with conical frustum model can predict the gauge length effect more accurately than the conventional Weibull distribution with cylindrical model.
\end{abstract}

Keywords: Weibull analysis; tensile strength; banana fiber; gauge length effect.

\section{INTRODUCTION}

Advances in science towards a sustainable future have been the highlight for most of the 21 st century. In regards to microscopic scale of engineering, there has been a shift of interest from synthetic fibers to natural fibers. Natural fibers have many advantages over synthetic fibers; they are lightweight, renewable, and of lower cost, additionally natural fibers possess the capability to provide similar, if not better reinforcement in composite materials [1-4].

Banana fibers tend to be an unused product of the banana tree and hence its usage will contribute to the renewability factor [5]. Kulkarni et al. [6] reported that the cellulose content of banana fiber is $65 \%$, whilst Oksman et al. [7] placed the cellulose content of banana fiber between $54 \%-63 \%$. This falls in line with the lower end of the cellulose content of jute fiber as Fidelis et al. [1] and Oksman et al. [7] placed the cellulose content of jute fibers between $61 \%-71.5 \%$ and $61 \%-72 \%$ respectively.

However, due to the climate and growing conditions of the natural fibers (depending on where the plant is grown) there may be a reduction in quality [8]. One main issue regarding natural fibers is that the tensile strengths of natural fiber cannot be 
predicted accurately due to various cross-sectional area throughout the fiber length. The variation in the cross-sectional area along with the fiber morphological flaws affect the inconsistencies in tensile strength values [9]. These flaws increase as the gauge length increases, and therefore reduce the tensile strength $[6,9]$.

In order to predict the tensile strength of the banana fibers, the Weibull statistical analysis is used. This analysis has been conducted on oil palm fiber by Sia et al. [10], bamboo fiber by Wang et al. [11, 12], palm leaf sheath fiber by Guo et al. [13], wool fiber by Zhang et al. [9], and jute fiber by Xia et al. [14] and Torres et al. [15]. However, no related work on strength prediction of banana fibers has been done by considering the variation of within fiber diameter variation. Although the diameter varies along the fiber Rao and Rao, cited in Venkateshwaran and Elayaperumal [16] reported that the banana fiber cross-section can be approximated to be circular. This claim is substantiated Mukhopadhyay et al. [17]. Likewise, for oil palm fiber [10], jute fiber $[14,15]$, and bamboo fiber $[11,12,18]$, the fiber cross-sections were approximated to be circular in shape.

\section{THEORETICAL BACKGROUND}

The simple Weibull distribution for fiber strength is as follows [19]:

$$
P_{f}=1-\exp \left[-\left(\frac{V}{V_{o}}\right)\left(\frac{\sigma_{f}}{\sigma_{o}}\right)^{m}\right]
$$

where $P_{f}$ refers to the probability of failure of the fiber, $V$ refers to the volume of the fiber, $V_{\mathrm{o}}$ refers to the reference volume, $\sigma_{f}$ indicates the strength at which the fiber fails, $\sigma_{\mathrm{o}}$ corresponds to the scale parameter and $m$ corresponds to the Weibull modulus. The average value of $\sigma f$ can be computed using the following equation:

$$
\bar{\sigma}_{f}=\sigma_{o}\left(\frac{V}{V_{o}}\right)^{-\frac{1}{m}} \Gamma\left(1+\frac{1}{m_{o}}\right)
$$

The average value of $\sigma_{f 1}$ together with $L_{1}$ and $m$ can be used to calculate $\sigma_{f 2}$ at its corresponding length $L_{2}$, which is the gauge length effect.

$$
\overline{\sigma_{f 2}}=\overline{\sigma_{f 1}}\left(\frac{L_{2}}{L_{1}}\right)^{-\frac{1}{m}}
$$

This equation is valid for constant fiber diameter. However, due to this equation incorporating only constant fiber diameter, the subsequent results obtained were reported to vary greatly in comparison to experimental values. Hence a modification to this conventional Weibull model was proposed by Gutans and Tamuzh [20] and Watson and Smith [21], in which the $-1 / m$ in Eq. (2) was changed to $\alpha / m, 0<\alpha<1$, where $\alpha$ is the shape parameter. Hence, the modified equations are as shown below.

$$
P_{f}=1-\exp \left[-\left(\frac{V}{V_{o}}\right)^{\alpha}\left(\frac{\sigma_{f}}{\sigma_{o}}\right)^{m}\right]
$$




$$
\overline{\sigma_{f 2}}=\overline{\sigma_{f 1}}\left(\frac{L_{2}}{L_{1}}\right)^{-\frac{\alpha}{m}}
$$

Although the shape parameter $\alpha$ represents the within fiber diameter variations Zhang et al. [9] stated that the exact physical meaning is not clear. In order to determine the value of $\alpha$, the value of $\alpha$ can be determined using the following equation.

$$
\ln \left(C V_{F D}\right)=\alpha \ln (L)+C+\varepsilon
$$

where $C V_{F D}$ is the average within fiber diameter variation of a certain number of samples, $\mathrm{L}$ is the gauge length, $\mathrm{C}$ is a constant and $\varepsilon$ is a random error. This equation corresponds to the graph in which $\mathrm{Y}=\ln (C V)$ and $\mathrm{X}=\ln (L)$. Hence $\alpha$ is the gradient of this graph.

Rearranging the modified Weibull distribution whilst taking $\ln$ of either side provides the following formula.

$$
\ln \left(-\ln \left(1-P_{f}\right)\right)-\alpha \ln \left(\frac{V}{V_{o}}\right)=m \ln \sigma_{f}-m \ln \sigma_{o}
$$

Thereafter, by plotting a graph of $f \ln \left(-\ln \left(1-P_{f}\right)\right)-\alpha \ln \left(\frac{V}{V_{o}}\right)$ against $\ln \sigma_{f}$, a linear graph is produced. Therefore, the gradient of the graph will be the modulus $m$, whilst $\sigma_{\mathrm{o}}$ is the intercept when $\ln \left(-\ln \left(1-P_{f}\right)\right)-\alpha \ln \left(\frac{V}{V_{o}}\right)=0$. The probability of failure $P_{f}$ can be determined using the equation shown below.

$$
P_{f}=\frac{i-0.3}{N+0.4}
$$

Where $i$ corresponds to rank of the respective ascending ordered data points and $\mathrm{N}$ is the number of samples or data points.

In this research, conventional and modified Weibull models will be applied to predict the banana fiber tensile strength. The modified model will be conducted via cylindrical model which incorporates the within fiber diameter variation and the conical frustum model which incorporates the within fiber conical frustum volume variation. The predicted strength will be expected more accurate by considering the variation of the conical frustum model as the model is closer to the geometry of the banana fiber.

\section{MATERIALS AND METHODS}

\section{Materials}

Banana fibers shown in Figure 1 were obtained from Venture Industries, Sri Lanka. The sodium hydroxide pellets were acquired from Swinburne University of Technology, Sarawak Campus. The sodium hydroxide pellets originated from the company UNICHEM. The physical and mechanical properties of the banana fibers are listed in Table.1. 
Table 1. Physical and mechanical properties of banana fibers [22].

\begin{tabular}{cc}
\hline Property & Range \\
\hline Diameter $(\mu \mathrm{m})$ & $105-210$ \\
Ultimate Strength $(\mathrm{MPa})$ & $198.9-300$ \\
Modulus Young $(\mathrm{GPa})$ & $6.6-13.7$ \\
Strain $(\%)$ & $1.93-3.27$ \\
\hline
\end{tabular}

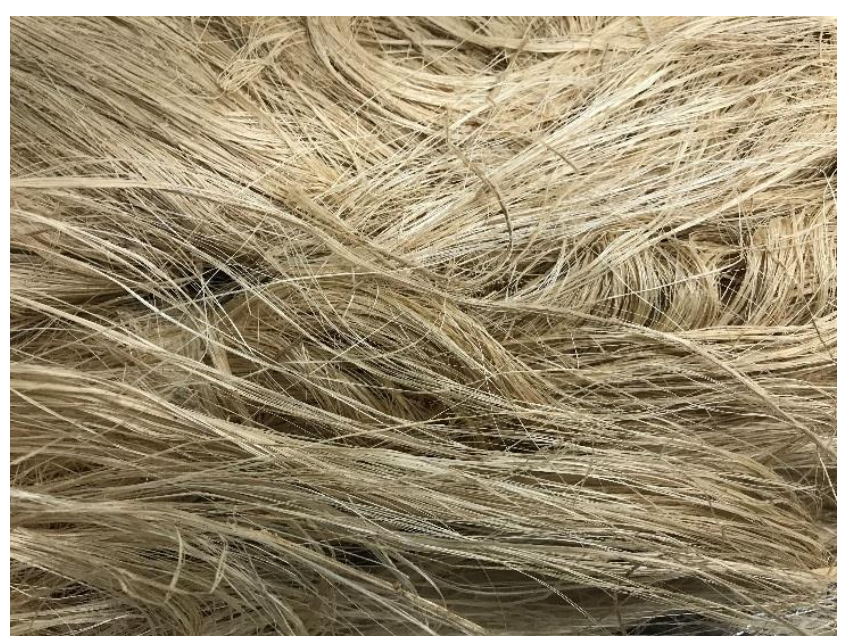

Figure 1. Banana fibers.

\section{Chemical Treatment}

Alkaline treatment was used to treat the banana fibers, wherein a $\mathrm{NaOH}$ percentage of $1 \%$ was used to soak the fibers for 30 minutes. Thereafter the banana fibers were washed thoroughly to ensure all excess sodium hydroxide and eventually result in a pH of 7 . Afterwards, the fibers were left to dry at room temperature for 24 hours.

\section{Fiber Diameter Scanning}

Once the epoxy cured, the fibers were observed along their gauge length using a Nikon Eclipse LV150 optical microscope. The scanned images were thereafter analysed using the software ImageJ and the fiber diameter was calculated at every $1 \mathrm{~mm}$ along the gauge lengths of $10 \mathrm{~mm}, 20 \mathrm{~mm}, 25 \mathrm{~mm}$, and $40 \mathrm{~mm}$.

\section{Specimen Preparation}

The specimens were prepared according to ASTM D3822-01. Lankem Epi-Fix epoxy hardener and resin was used to fasten the two ends of the fibers onto the cardboard. The schematic of specimen design is illustrated in Figure 2. 


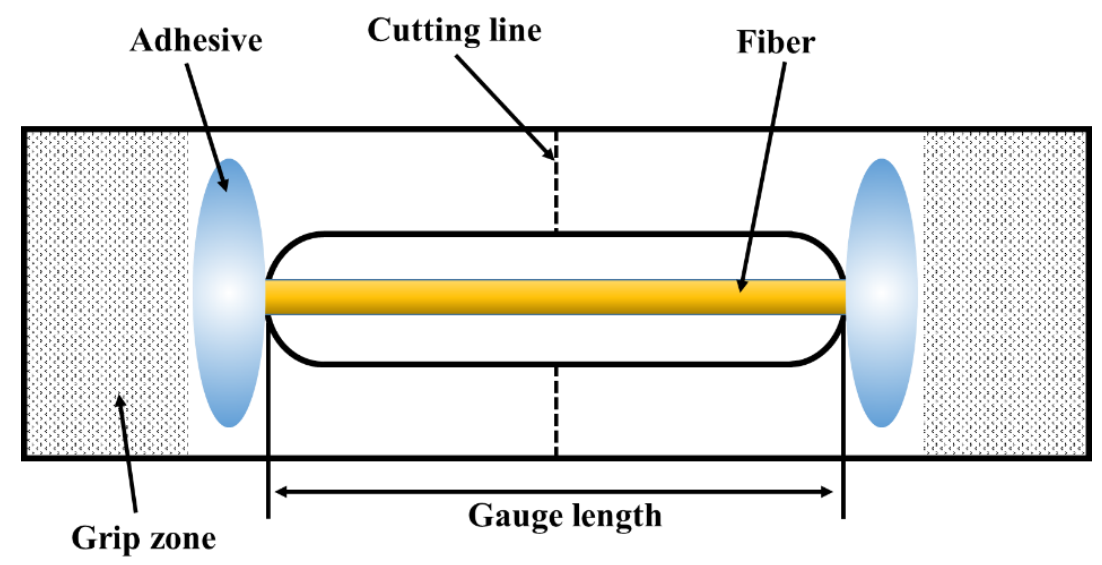

Figure 2. Schematic of the specimen.

\section{Tensile Testing}

Tensile testing was conducted using the Universal Testing Machine Instron 5569. The crosshead speed was set to $1 \mathrm{~mm} / \mathrm{min}$ and the test was carried out under controlled temperature and humidity of $20^{\circ} \mathrm{C} \pm 2{ }^{\circ} \mathrm{C}$ and $65 \pm 2 \% \mathrm{RH}$ respectively. The tensile strengths of the fibers were evaluated at $10 \mathrm{~mm}, 20 \mathrm{~mm}, 25 \mathrm{~mm}$ and $40 \mathrm{~mm}$ gauge lengths. Failure strengths of 20 successful specimens were obtained for each of the four gauge lengths.

\section{RESULTS AND DISCUSSION}

\section{Fiber Morphology}

The scanned images of an untreated and treated fiber is shown in Figure 3(a) and 3(b). Treatment with alkali solution removes waxes and dirt situated on the fiber surface and this can be observed in the two Figures shown in Figure 3. Many studies also revealed that the impurities in natural fibers can be cleaned out by alkali treatment [23-26]. In this study, the fiber is pre-treated with alkaline treatment is because the parameter $\alpha$ is dependent on the within-fiber diameter variation along the fiber length. Therefore, a clean fiber surface can improve the accuracy of the statistical analysis.

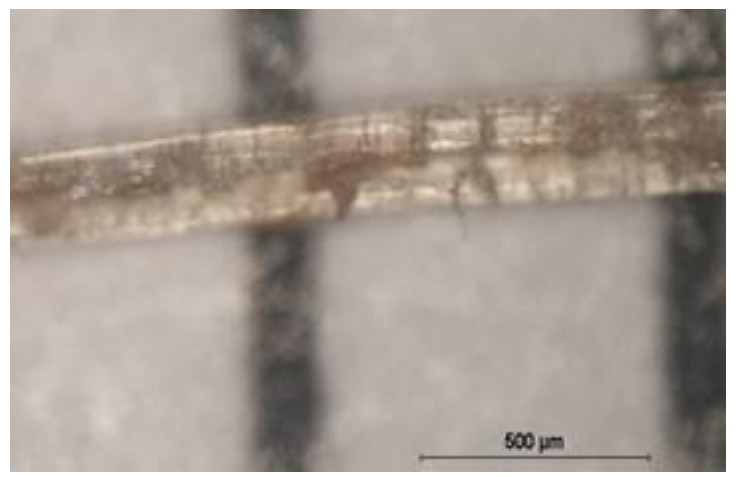

Untreated banana fiber

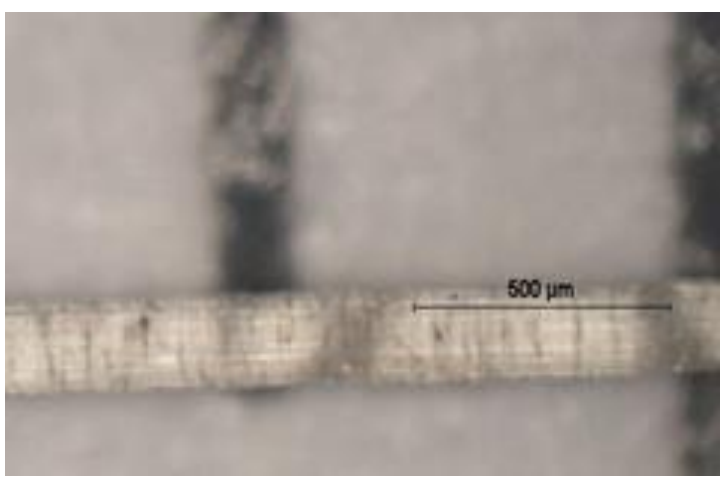

Alkaline treated banana

Figure 3. Untreated banana fiber and Alkaline treated banana fiber

(Magnification: 50X) 


\section{Banana Fiber Strength}

20 successful failure strengths for each gauge length of $10 \mathrm{~mm}, 20 \mathrm{~mm}, 25 \mathrm{~mm}$, and 40 $\mathrm{mm}$ are shown in Table 2. The mean diameter shown in Table 2 refers to the average diameter of the 20 successful fibers for each gauge length. The $C V_{\mathrm{FD}}$ (Fiber diameter Cylindrical model) and $C V_{\mathrm{FV}}$ (Frustum volume - Conical Frustum model) shown in Table 2 are the respective coefficient of variance of the 20 specimens. As the $C V_{\mathrm{FD}}$ and $C V_{\mathrm{FV}}$ increase the fiber failure strength decreases, this is observed even though there is an anomaly with $40 \mathrm{~mm}$ data where the fiber failure strength is lower at $25 \mathrm{~mm}$ when compared with fiber failure strength at $40 \mathrm{~mm}$. This anomaly can be due to a larger number of flaws in the $25 \mathrm{~mm}$ fibers than the $40 \mathrm{~mm}$ fibers.

Table 2. Fiber properties and respective CV.

\begin{tabular}{ccccc}
\hline $\begin{array}{c}\text { Gauge Length } \\
(\mathrm{mm})\end{array}$ & $\begin{array}{c}\text { Mean Diameter } \\
(\mu \mathrm{m})\end{array}$ & $\begin{array}{c}C V_{\mathrm{FD}} \\
(\%)\end{array}$ & $\begin{array}{c}C V_{\mathrm{FV}} \\
(\%)\end{array}$ & $\begin{array}{c}\text { Failure Strength } \\
(\mathrm{MPa})\end{array}$ \\
\hline 10 & 165.00 & 7.71 & 11.47 & 286.91 \\
20 & 141.07 & 8.75 & 14.42 & 286.76 \\
25 & 162.23 & 9.59 & 17.06 & 210.83 \\
40 & 152.74 & 10.83 & 19.32 & 239.80 \\
\hline
\end{tabular}

\section{Weibull Distribution Analysis}

Table 2 displays that increase in $C V_{\mathrm{FD}}$ and $C V_{\mathrm{FV}}$ occurs exponentially as the gauge length increase. This was observed in wool [9] , oil palm fiber [10], palm leaf sheath fiber [13], and jute fiber [14]. The increase in $C V_{\mathrm{FD}}$ and $C V_{\mathrm{FV}}$ is known to cause inconsistencies in tensile strength values as stated in the introduction. From the data in Table 2, it can be verified that fiber morphological flaws, within fiber diameter variation and within fiber conical frustum volume variation affect the fiber's failure strength properties by increasing the gauge length.

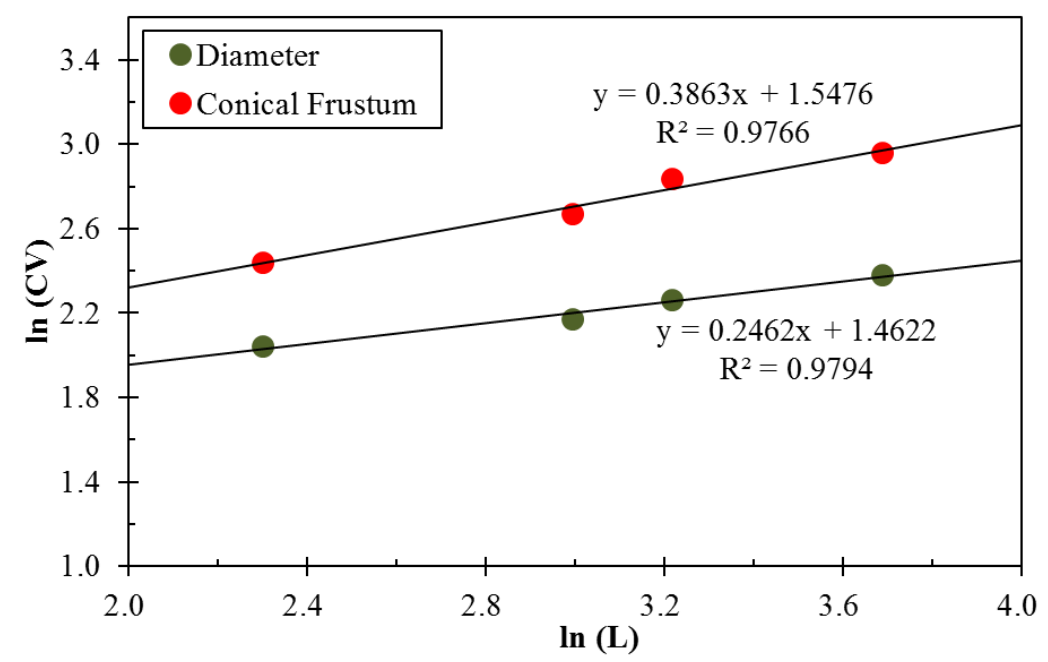

Figure 4. Relationship between CV and gauge length.

To investigate the gauge length effect it was necessary to obtain the shape parameter $\alpha$, this was achieved using Eq. (6) which Zhang et al. [9] proposed. figu 4 represents the graph of $\ln \left(C V_{F D}\right)=\alpha \ln (L)+C+\varepsilon$. Therefore, the shape parameter $\alpha$ (The slope of each trend line) for the cylindrical model is 0.2642 and for the Conical 
Frustum model is 0.3863 . The correlation of logarithm, $\mathrm{R}^{2}$ is $97.94 \%$ and $97.66 \%$, is high for both models and hence the regression line represents the experimental data effectively. Figure 5 exhibits the Weibull plots based on Eq. (7). Each gauge length includes two Weibull plots; cylindrical model and conical frustum model. The correlation of logarithm for all the graphs range from $92.79 \%$ to $96.72 \%$.
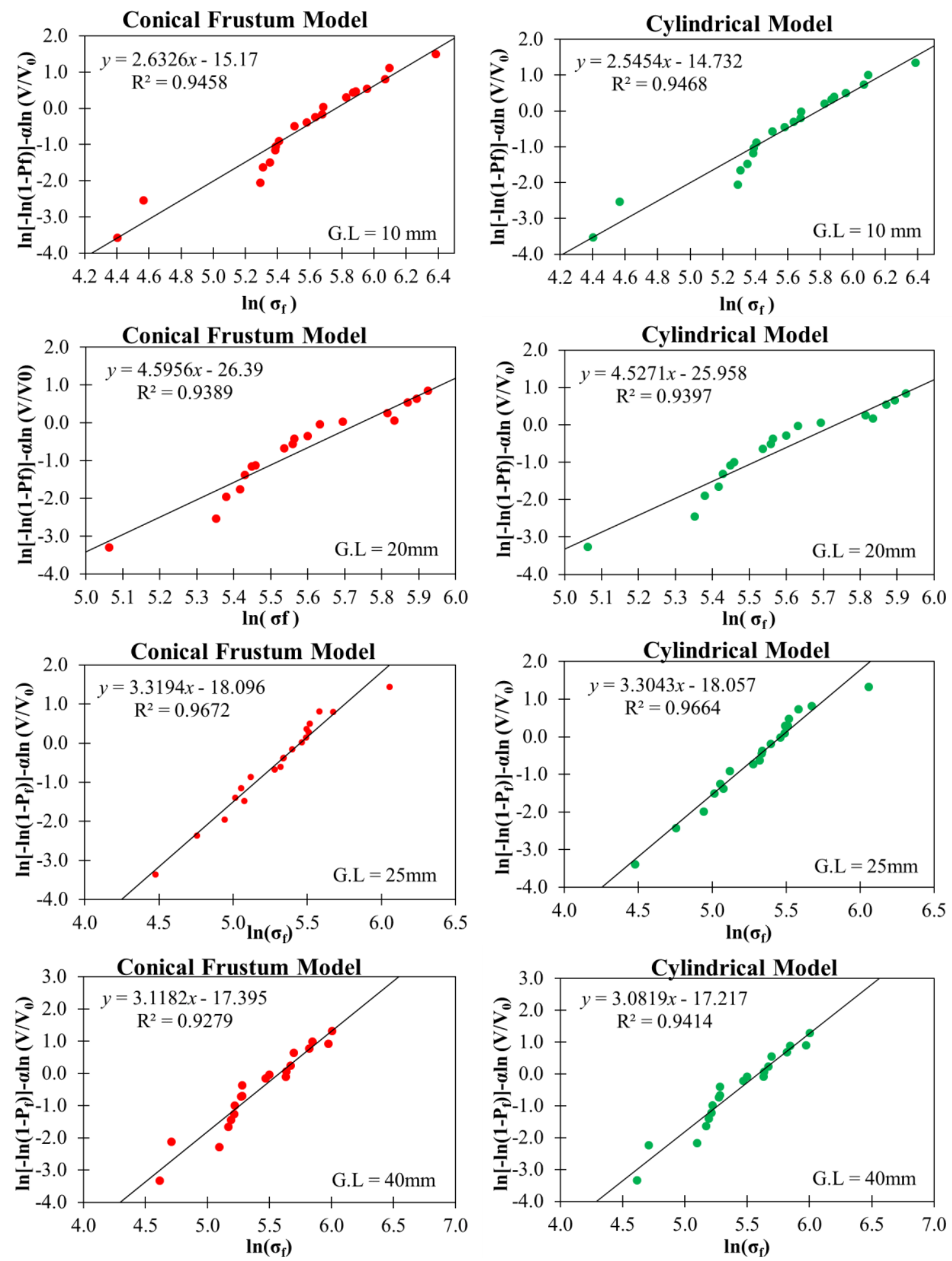

Figure 5. Modified Weibull plots for fiber strength at each gauge length. 


\section{Prediction of Failure Strength}

In order to predict the failure strength, the Weibull modulus, $m$ and scale parameter, $\sigma_{\mathrm{o}}$ for each gauge length is required. As mentioned in the theoretical background, this is obtained through the slope $(m)$ and intercept $\left(\sigma_{0}\right)$ of the Modified Weibull plots in Figure 5. These data are depicted in Table 3.

Table 3. The modified Weibull parameters.

\begin{tabular}{ccccc}
\hline & \multicolumn{2}{c}{ Weibull Modulus $(m)$} & \multicolumn{2}{c}{ Scale Parameter $\left(\sigma_{0}\right)$} \\
\cline { 2 - 5 } & & Conical & Conical \\
Gauge Length $(\mathrm{mm})$ & Cylindrical & Frustum & Cylindrical & Frustum \\
\hline 10 & 2.55 & 2.63 & 326.21 & 318.07 \\
20 & 4.53 & 4.60 & 309.13 & 313.53 \\
25 & 3.30 & 3.32 & 236.19 & 233.11 \\
40 & 3.08 & 3.12 & 266.77 & 264.69 \\
\hline
\end{tabular}

The higher the Weibull modulus the lower the variability in the strength and vice versa [1], moreover Wang et al. [11] stated the Weibull modulus, $m$ of natural fiber are generally falls in the range of 1 to 6 . The results in Table 3 fall within this range, likewise does jute [14], and oil palm fiber [10]. The Weibull modulus in Table 3 for the $10 \mathrm{~mm}$ gauge length does not follow the gauge length effect, where the smallest length should have the highest value of $\mathrm{m}$, nevertheless the modulus' starts to decrease with gauge length from $20 \mathrm{~mm}$ to $40 \mathrm{~mm}$, thereby following the gauge length effect. This anomaly is due to the failure strength having a larger variation between maximum and minimum values. This is evident in Figure 5, $10 \mathrm{~mm}$ gauge length plots. Wang et al. [11] reported similarly for bamboo fibers and stated the cause to be experimental uncertainties. However, the scale parameter, which denotes the characteristic strength, follows the gauge length effect wherein it reduces as the gauge length increases. This is due to a higher number of flaws being present at a longer length. Similar behaviour was observed with palm leaf sheath fiber [13], jute fiber [14], and oil palm fiber [10].

The predictions presented in Figure 6 were calculated using Eq. (3) and (5) for the conventional Weibull model, and Cylindrical and Conical Frustum models respectively. The prediction calculations were based on the $10 \mathrm{~mm}$ and $40 \mathrm{~mm}$ experimental results. The results are plotted based on $95 \%$ confidence intervals.

Figure 6(a) and 6(b) represents the predictions based on $10 \mathrm{~mm}$ and $40 \mathrm{~mm}$ gauge lengths respectively. In both plots, it is evident that both modified models present better predictions in comparison to the conventional model. Furthermore, amongst the two modified Weibull models, in Figure 6(a) and 6(b), the Conical Frustum model which incorporates the within fiber Conical Frustum volume variations can predict the gauge length effect more precisely than the Cylindrical model which incorporates the within fiber diameter variations. 


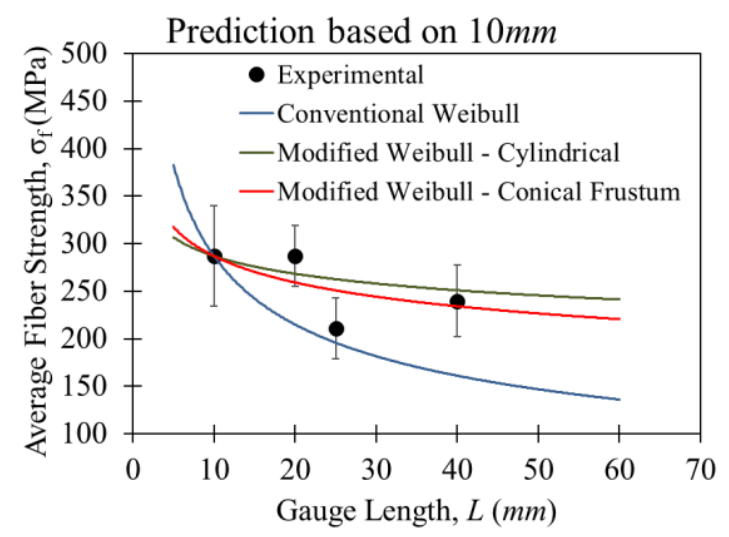

(a)

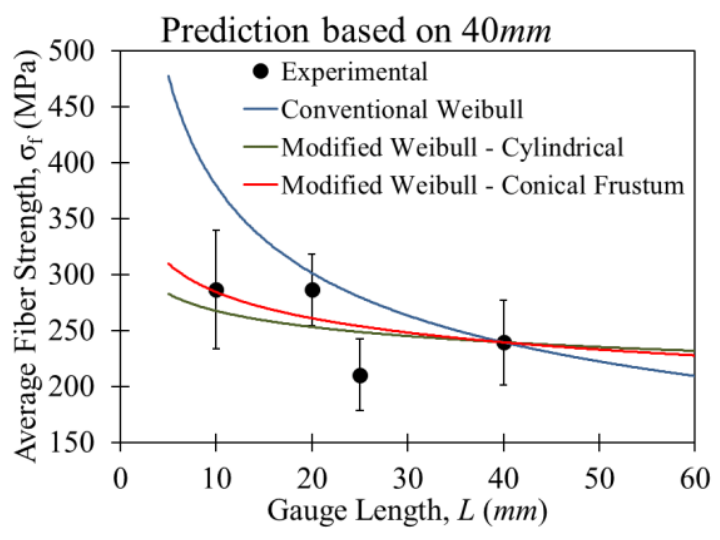

(b)

Figure 6. Predictions based on (a)10 mm and (b) $40 \mathrm{~mm}$ gauge length (Experimental results are plotted at $95 \%$ confidence intervals).

\section{CONCLUSIONS}

This research evaluated the effect of varying cross-sectional area on the failure strength of banana fibers at gauge lengths of $10 \mathrm{~mm}, 20 \mathrm{~mm}, 25 \mathrm{~mm}$, and $40 \mathrm{~mm}$. It can be concluded that the fiber strength decreases as the gauge length increases; this is brought about due to the number of flaws increasing along with the varying cross-sectional area. The modified Weibull model was deemed more accurate compared to the conventional Weibull model. The predicted strength by incorporating the within fiber conical frustum volume variation shows the best result in fiber strength prediction.

\section{REFERENCES}

[1] Alves Fidelis ME, Pereira TVC, Gomes OdFM, de Andrade Silva F, Toledo Filho RD. The effect of fiber morphology on the tensile strength of natural fibers. Journal of Materials Research and Technology. 2013;2:149-57.

[2] Pickering KL, Efendy MGA, Le TM. A review of recent developments in natural fibre composites and their mechanical performance. Composites Part A: Applied Science and Manufacturing. 2016;83:98-112.

[3] Joshi SV, Drzal LT, Mohanty AK, Arora S. Are natural fiber composites environmentally superior to glass fiber reinforced composites? Composites Part A: Applied Science and Manufacturing. 2004;35:371-76.

[4] Mohammed L, Ansari MNM, Pua G, Jawaid M, Islam MS. A Review on Natural Fiber Reinforced Polymer Composite and Its Applications. International Journal of Polymer Science. 2015;2015:15.

[5] Ramesh M, Atreya TSA, Aswin US, Eashwar H, Deepa C. Processing and Mechanical Property Evaluation of Banana Fiber Reinforced Polymer Composites. Procedia Engineering. 2014;97:563-72. 
[6] Kulkarni AG, Satyanarayana KG, Rohatgi PK, Vijayan K. Mechanical properties of banana fibres (Musa sepientum). Journal of Materials Science. 1983;18:2290-96.

[7] Oksman K, Mathew AP, Långström R, Nyström B, Joseph K. The influence of fibre microstructure on fibre breakage and mechanical properties of natural fibre reinforced polypropylene. Composites Science and Technology. 2009;69:184753.

[8] Hu W, Ton-That MT, Perrin-Sarazin F, Denault J. An improved method for single fiber tensile test of natural fibers. Polymer Engineering \& Science. 2009;50:819-25.

[9] Zhang Y, Wang X, Pan N, Postle R. Weibull analysis of the tensile behavior of fibers with geometrical irregularities. Journal of Materials Science. 2002;37:1401-06.

[10] Sia CV, Nakai Y, Shiozawa D, Ohtani H. Statistical analysis of the tensile strength of treated oil palm fiber by utilisation of Weibull distribution model. Open Journal of Composite Materials. 2014;4:72-77.

[11] Wang F, Shao J. Modified Weibull Distribution for Analyzing the Tensile Strength of Bamboo Fibers. Polymers. 2014;6:3005.

[12] Wang F, Shao J, Li X. Statistics on the fracture strength of bamboo fibers. Polymer Composites. 2015;37:221-28.

[13] Guo M, Zhang TH, Chen BW, Cheng L. Tensile strength analysis of palm leaf sheath fiber with Weibull distribution. Composites Part A: Applied Science and Manufacturing. 2014;62:45-51.

[14] Xia ZP, Yu JY, Cheng LD, Liu LF, Wang WM. Study on the breaking strength of jute fibres using modified Weibull distribution. Composites Part A: Applied Science and Manufacturing. 2009;40:54-59.

[15] Torres JP, Vandi LJ, Veidt M, Heiztmann MT. Statistical data for the tensile properties of natural fibre composites. Data in Brief. 2017;12:222-26.

[16] Venkateshwaran N, Elayaperumal A. Banana Fiber Reinforced Polymer Composites - A Review. Journal of Reinforced Plastics and Composites. 2010;29:2387-96.

[17] Samrat M, Raul F, Vijay S. Variability of Tensile Properties of Fibers from Pseudostem of Banana Plant. Textile Research Journal. 2009;79:387-93.

[18] Monteiro SN, Margem FM, Braga FdO, Luz FSd, Simonassi NT. Weibull analysis of the tensile strength dependence with fiber diameter of giant bamboo. Journal of Materials Research and Technology. 2017;6:317-22.

[19] McCool JI. Using the Weibull Distribution: Reliability, Modeling, and Inference. Wiley Series in Probability and Statistics. New York: John Wiley and Sons; 2012.

[20] Gutans YA, Tamuzh VP. Probability analysis of the fracture of unidirectional hybrid composites in tension. Mechanics of Composite Materials. 1986;22:32431.

[21] Watson AS, Smith RL. An examination of statistical theories for fibrous materials in the light of experimental data. Journal of Materials Science. 1985;20:3260-70.

[22] Mejía Osorio JC, Rodríguez Baracaldo R, Olaya Florez JJ. The influence of alkali treatment on banana fibre's mechanical properties. 2012. 2012;32:5. 
[23] Gassan J, Bledzki Andrzej K. Alkali treatment of jute fibers: Relationship between structure and mechanical properties. Journal of Applied Polymer Science. 1999;71:623-29.

[24] Li X, Tabil LG, Panigrahi S. Chemical Treatments of Natural Fiber for Use in Natural Fiber-Reinforced Composites: A Review. Journal of Polymers and the Environment. 2007;15:25-33.

[25] Moshiul Alam AKM, Beg MDH, Reddy Prasad DM, Khan MR, Mina MF. Structures and performances of simultaneous ultrasound and alkali treated oil palm empty fruit bunch fiber reinforced poly(lactic acid) composites. Composites Part A: Applied Science and Manufacturing. 2012;43:1921-29.

[26] Sreekala MS, Kumaran MG, Joseph R, Thomas S. Stress-relaxation behaviour in composites based on short oil-palm fibres and phenol formaldehyde resin. Composites Science and Technology. 2001;61:1175-88. 